\title{
Fusion hindrance of heavy ions: role of the neck
}

\author{
David Boilley* and Hongliang Lü \\ GANIL, CEA/DSM-CNRS/IN2P3, BP 55027, F-14076 Caen cedex 5, France and \\ Université de Caen Basse-Normandie, BP 5186, F-14032 Caen Cedex, France \\ Caiwan Shen \\ School of Science, Huzhou Tearchers College, Huzhou, 313000 Zhejiang, P.R. China \\ Yasuhisa Abe \\ Research Center for Nuclear Physics, Osaka University, \\ 10-1 Mihogaoka, Ibaraki, 567-0047 Osaka, Japan \\ Bertrand G. Giraud \\ Institut Physique Théorique, CEA/DSM, Centre d'Etudes-Saclay, Gif-sur-Yvette, F-91191, France
}

\begin{abstract}
Fusion of heavy ions is largely hindered because of the appearance of an inner barrier between the contact point of the two colliding nuclei and the compound nucleus. But there are still quantitative ambiguities on the size of the barrier and on the role of the dissipation. In this paper we stress the importance of the neck of the composite system on the hindrance of the fusion of heavy nuclei. We show that the "denecking" process is very quick compared to the other collective degrees of freedom as the relative distance. This behavior of the neck will change the potential seen by the relative distance on the way to fusion and its effective initial value through a dynamical coupling. Both effects contribute to the hindrance of fusion.
\end{abstract}

PACS numbers: 25.70.Jj,24.10.-i,24.10.Pa

\section{INTRODUCTION}

Fusion of heavy ions is largely hindered by comparison to what is observed for lighter systems. This fact that has been observed experimentally since many years ago $[1,2]$ is nowadays qualitatively understood: after crossing the Coulomb barrier, the fusing system at contact have to overcome a second barrier under strong dissipation. This inner barrier does not exist for lighter systems that directly reach the compound state after crossing the Coulomb barrier. Such an interpretation is commonly accepted [3-16], but there are still quantitative ambiguities on the dynamics of the fusion mechanism and predictions might not be reliable.

Experimentally, it is very difficult to distinguish between the fusion-fission events that have reached the compound state and the quasi-fission ones that re-separate after crossing the Coulomb barrier. This leads to a lack of reliable data on fusion cross-sections that could assess the models. The assessment of the various models used to describe the complete fusion process is then one of the main challenges of the field. For a recent review, see e.g. Ref. [17].

Theoretically, the fusion process is divided into two steps corresponding to the crossing of the two consecutive barriers [8]. For the Coulomb barrier, an extrapolation of the simple models used for lighter systems without hindrance is sometime used $[15,18,19]$ and we can rely

\footnotetext{
*boilley@ganil.fr
}

on the experimental capture cross sections. Actually, the dissipation process cannot be neglected during this step for heavy ions because of the large Coulomb field. It leads to a partial explanation of the fusion hindrance [20, 21]. Coupled channel codes can nicely reproduce experimental data for light systems, even far below the barrier. But, the inclusion of some dissipation mechanisms that would be necessary for energies over the barrier is still under development [22-24].

The main contribution to the fusion hindrance observed in heavy ions is due to the second step, consisting of a diffusion process over an inner potential barrier. The main features of the dynamics of this so-called formation phase are well understood: most of the models are based on stochastic equations [6-16]. This is justified by the fact that the intrinsic degrees of freedom had enough time to thermalize during the crossing of the Coulomb barrier. But ambiguities remain on the size of the barrier and the strength of the dissipation.

Another difficulty arises from the fact that the two steps of the fusion process cannot be treated with a single formalism. We have to deal with a parameterization for the two-body system crossing the Coulomb barrier and with another one for the composite from the contact point to the compound shape. The treatment of the connection between the two decriptions is a delicate problem that can change the final results.

As we will show in this paper, the fusion process is very sensitive to the treatment of the evolution of the neck between the two colliding nuclei at contact, because the size of the barrier that has to be overcome strongly depends on it. See Fig. 1. The formation probability 
depends exponentially on this barrier and depending on the treatment, the final fusion cross section can differ by orders of magnitude. It is important to note that some of the previous studies on the effect of the neck on the fusion process are related to the Coulomb barrier [25, 26]. Here, we focus our work on the inner barrier.

In some studies, the neck was supposed to be frozen at a given value, that was arbitrarily fixed $[13,14]$ or adjusted to fit some experimental fusion cross sections [8]. Refs. [27, 28], claim that the previous hypothesis is correct: a dynamical study suggests that the neck does not evolves much during the fusion process. In some other works [10-12], the inner potential barriers are evaluated by minimization with respect to the neck. "Denecking" process, i.e. disappearance of the cleft between the two nuclei at contact, is also very fast in the fusion trajectory chosen in $[29,30]$ to calculate the potential barriers. In preliminary versions of this work reported in conferences [31-34] and in Refs. [35, 36], it is shown on dynamical arguments that it is correct. Finally, in $[15,16]$ the neck degree of freedom is not mentioned.

The previous models are based on macroscopic approaches. There are also tentatives to describe the fusion process with microscopic models based on mean field theory or molecular dynamics. But there are very few connections between microscopic and macroscopic approaches. In Ref. [21], macroscopic parameters were extracted from a mean field approach, but this study is limited to the Coulomb barrier. To our knowledge, Ref. [35] is one the rare study of the dynamics of the neck studied from a microscopic approach based on molecular dynamics.

How is the neck evolving during the fusion process? What is the most suitable value of this variable? What does it mean for the other collective variables? We have to answer to these questions in order to understand the fusion mechanism.

\section{SIMPLIFIED NECK DYNAMICS}

The neck parameter $\varepsilon$ is related to the cleft between the two touching nuclei. $\varepsilon=1$ corresponds to two hard touching spheres and $\varepsilon=0$ to the absence of cleft. More precisely, in our calculations, the neck parameter is taken from the Two-Center parameterization [37] and is defined by the potential shape of the interacting nuclei. The dissipation is calculated with the wall-and-window formalism [38, 39].

The dynamics of the formation phase is frequently based on coupled stochastic equations [6-16]. The other two variables are the relative distance between the centers of mass of the two nuclei and the mass-asymmetry of the colliding system. These collective degrees of freedom are connected through the Liquid Drop Model (LDM) potential, the collective inertia and friction. But as a first step, we will study the dynamics of the neck separately.

As for the LDM potential, it turns out that it is al-
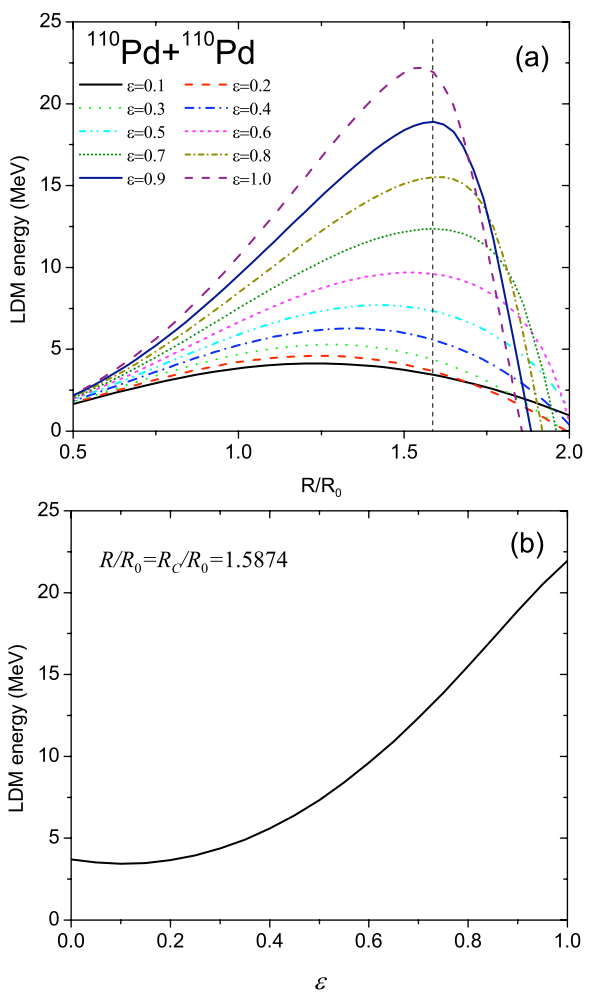

FIG. 1. [Color online] Top: LDM potential energy of the system ${ }^{110} \mathrm{Pd}+{ }^{110} \mathrm{Pd}$ as a function of the elongation coordinate for various neck parameters. $\varepsilon$ is the neck parameter and $R_{0}$ the radius of the compound nucleus. The vertical dashed line on the top panel represents the contact position. Bottom: LDM potential energy as a function of the neck parameter along the contact line.

most linear in the neck parameter at contact. See Fig. 1. Then, for a simple analysis of the neck dynamics, we solve analytically the Smoluchowski equation with a linear potential,

$$
\frac{\partial N(\varepsilon, t)}{\partial t}=C \frac{\partial N(\varepsilon, t)}{\partial \varepsilon}+D \frac{\partial^{2} N(\varepsilon, t)}{\partial \varepsilon^{2}}
$$

where $C=f / \gamma$ and the diffusion coefficient $D=k T / \gamma$. Here $f$ is a constant parameter such as $V(\varepsilon)=f \cdot \varepsilon$ and $\gamma$ is the friction coefficient. Since the neck parameter is limited to the $[0,1]$ interval, we will add two reflective boundaries in $\varepsilon=0$ and $\varepsilon=1$.

With a single reflective boundary in $\varepsilon=0$ and an initial distribution taken as $N_{1}(\varepsilon, 0)=\delta\left(\varepsilon-\varepsilon_{0}\right)$, the Smoluchowski equation (1) was solved in Refs. [40-42],

$$
\begin{aligned}
N_{1}(\varepsilon, t)= & \frac{1}{\sqrt{4 \pi D t}} \exp \left[-\frac{C}{2 D}\left(\varepsilon-\varepsilon_{0}\right)-\frac{C^{2} t}{4 D}\right] \\
& \times\left(\exp \left[-\frac{\left(\varepsilon-\varepsilon_{0}\right)^{2}}{4 D t}\right]+\exp \left[-\frac{\left(\varepsilon+\varepsilon_{0}\right)^{2}}{4 D t}\right]\right) \\
& +\frac{C}{2 D} \exp \left[-\frac{C \varepsilon}{D}\right] \cdot \operatorname{erfc}\left[\frac{\varepsilon+\varepsilon_{0}-C t}{2 \sqrt{D t}}\right] \cdot
\end{aligned}
$$

For large times, this expression becomes a Boltzmann dis- 
tribution. The average value of the neck was calculated in Ref. [43],

$$
\begin{aligned}
\langle\varepsilon(t)\rangle= & \varepsilon_{0}-C t+\frac{1}{2}\left(\frac{D}{C}-\varepsilon_{0}+C t\right) \operatorname{erfc}\left[\frac{\varepsilon_{0}-C t}{\sqrt{4 D t}}\right] \\
& -\frac{D}{2 C} \exp \left[\frac{C \varepsilon_{0}}{D}\right] \operatorname{erfc}\left[\frac{\varepsilon_{0}+C t}{\sqrt{4 D t}}\right] \\
& +\sqrt{\frac{D t}{\pi}} \exp \left[-\frac{\left(\varepsilon_{0}-C t\right)^{2}}{4 D t}\right] .
\end{aligned}
$$

We also solved the problem with the two reflective boundaries, following the method of Refs. [41, 44] and get,

$$
\begin{aligned}
N_{2}(\varepsilon, t)= & \frac{C}{D} \frac{\exp \left[-\frac{C \varepsilon}{D}\right]}{1-\exp \left[-\frac{C a}{D}\right]} \\
& +\exp \left[-\frac{C}{2 D}\left(\varepsilon-\varepsilon_{0}\right)-\frac{C^{2} t}{4 D}\right] \\
& \times \sum_{k=1}^{\infty}\left\{\exp \left[-\frac{k^{2} \pi^{2} D}{a^{2}} t\right] \frac{2}{a\left(1+\frac{C^{2} a^{2}}{4 D^{2} k^{2} \pi^{2}}\right)}\right. \\
& \left(\cos \left(\frac{k \pi \varepsilon}{a}\right)-\frac{C}{2 D} \frac{a}{k \pi} \sin \left(\frac{k \pi \varepsilon}{a}\right)\right) \\
& \left.\left(\cos \left(\frac{k \pi \varepsilon_{0}}{a}\right)-\frac{C}{2 D} \frac{a}{k \pi} \sin \left(\frac{k \pi \varepsilon_{0}}{a}\right)\right)\right\} .
\end{aligned}
$$

For the sake of generality, we denote the position of the second reflective boundary by $a$. The average value of the neck as a function of time can be obtained in a similar way,

$$
\begin{aligned}
\langle\varepsilon(t)\rangle= & \frac{D}{C}+\frac{a}{1-\exp \left[\frac{C a}{D}\right]} \\
& +32 a \pi^{2} D^{4} \exp \left[\frac{C}{2 D} \varepsilon_{0}-\frac{C^{2} t}{4 D}\right] \\
& \times \sum_{k=1}^{\infty}\left\{\exp \left[-\frac{k^{2} \pi^{2} D}{a^{2}} t\right] \frac{k^{2}}{\left(a^{2} C^{2}+4 D^{2} k^{2} \pi^{2}\right)^{2}}\right. \\
& \left(\cos \left(\frac{k \pi \varepsilon_{0}}{a}\right)-\frac{C}{2 D} \frac{a}{k \pi} \sin \left(\frac{k \pi \varepsilon_{0}}{a}\right)\right) \\
& \left.\left((-1)^{k} \exp \left[-\frac{C a}{2 D}\right]-1\right)\right\}
\end{aligned}
$$

In Fig. 2, we plot the neck distribution and its average value as a function of time for the two cases. With such a strong slope of the potential, the reflecting wall in $a$ does not play any role. If we arbitrarily decrease this slope, the curves corresponding to the two situations differ in a clear manner.

It appears that the neck evolves very quickly to its asymptotic distribution for both cases. For small times, the average value of the neck is approximately

$$
\langle\varepsilon(t)\rangle \simeq \varepsilon_{0}-C t
$$

There is an ambiguity on the initial value of the neck parameter since the denecking process might already start
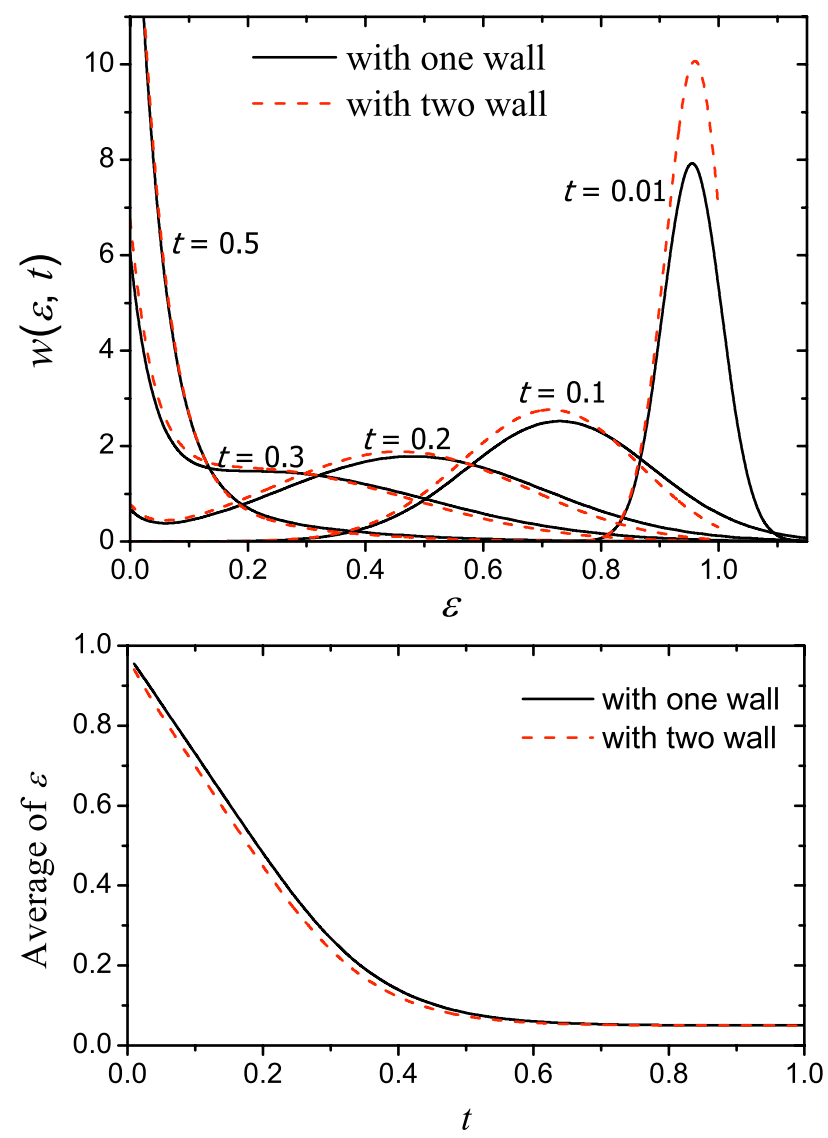

FIG. 2. [color online] Neck distribution and its average value as a function of time. The solid line corresponds to the case with one reflecting wall in $\varepsilon=0$ and the dashed one to the case with two reflective walls, in $\varepsilon=0$ and 1 . Here $C=2.5 \mathrm{MeV} / \hbar$ and $D=0.125 \mathrm{MeV} / \hbar$, which are typical values. Time is indicated in $\hbar / \mathrm{MeV}$. Here, the initial value of the neck is arbitrarily chosen as $\varepsilon_{0}=0.95$.

before crossing the Coulomb barrier [25, 26]. With the extreme hypothesis that the initial neck parameter is close to one, the time scale to reach small values for the neck parameter is then of the order of $1 / C=\gamma / f$. Typical values of $C$ are ranging from 1.1 to $2.7 \mathrm{MeV} / \hbar$.

The time scale of the fusion process along the relative distance was evaluated with a similar approach in Ref. [45]. For a diffusive process corresponding to the actual situation, the typical time to overcome the potential barrier is about one order of magnitude longer. Then, the time scale of the evolution of the neck is far shorter than the radial one and we can, as a first approximation, consider that the neck is completely thermalized during the second stage of the fusion process.

Actually, the potential shown in Fig. 1 is not linear for small values of $\varepsilon$. Unfortunately, analytical solutions are not available for higher order potentials with reflective boundaries. But, the linear approximation is valid for the initial values of the neck. A dynamical study with a more realistic potential will not change the characteristic time for the neck to reach small values. It will only affect 
the final thermal distribution.

Ref. [35] shows the time evolution of the neck calculated with a microscopic model and it appears that it is also shorter than the time characterizing the evolution of the relative distance. The velocity is peaked as function of time and vanishes on a time scale of the order of $100 \mathrm{fm} / \mathrm{c}$ or $0.5 \hbar / \mathrm{MeV}$, which is similar to our result. Our conclusion differs from the one of Refs. [27, 28] that claim that the neck is frozen around a value of about 0.7 , but is in agreement with the hypothesis done in Refs. [10-12, 29, 30].

Our analysis gives a quantitative confirmation of the discussion of Ref. [12]: the neck degree of freedom is quickly drifted by a strong potential slope, which is due to the large surface energy gain of the denecking process. With typical values of the potential for heavy nuclei, the thermalized neck parameter is very small, around or lower than 0.1 .

\section{APPEARANCE OF THE HINDRANCE}

The size and the location of the inner barrier along the relative distance that is calculated with the LDM are very sensitive to the neck. See Fig. 1. Depending on the relative position of this barrier to the contact point of the two colliding nuclei, the fusion will be hindered or not. Then, the experimental appearance of the hindrance should give some constraints on the location of the inner barrier and then the size of the neck $[9,33]$.

For symmetric reactions, the large hindrance phenomenon appears somewhere between the ${ }^{100} \mathrm{Mo}+$ ${ }^{100} \mathrm{Mo}$ and the ${ }^{110} \mathrm{Pd}+{ }^{110} \mathrm{Pd}$ systems [2]. Then the overlap of the inner barrier and the contact point should occur between these two systems if one considers the contact point as the injection point of the formation process. For the ${ }^{100} \mathrm{Mo}+{ }^{100}$ Mo system, the LDM potential landscape calculated within the two-center parameterization [37] is plotted in Fig. 3. It can be seen that the contact point is beyond this barrier, whatever the neck parameter. Then, after contact, the composite system is driven to the compound shape without hindrance. The situation differs for the ${ }^{110} \mathrm{Pd}+{ }^{110} \mathrm{Pd}$ system, see Figs. 1 and 3 . For small values of the neck $\varepsilon$, there is a large barrier between the contact point and the compound shape. For larger values of $\varepsilon$, the contact position is closer to the edge of the potential map. Then the neck parameter has to be smaller than 0.6 to explain the large hindrance of the fusion that is experimentally observed.

Although data are missing on symmetric systems between ${ }^{100} \mathrm{Mo}+{ }^{100} \mathrm{Mo}$ and ${ }^{110} \mathrm{Pd}+{ }^{110} \mathrm{Pd}$ to have a more precise analysis, this simple argument is in favor of our claim that the neck parameter should be small when the system crosses the inner barrier. The analysis presented here is limited to symmetric reactions. We did a systematic analysis of the border between hindered and nonhindered reactions that confirm the fact that the neck should disappear quickly $[9,46]$.
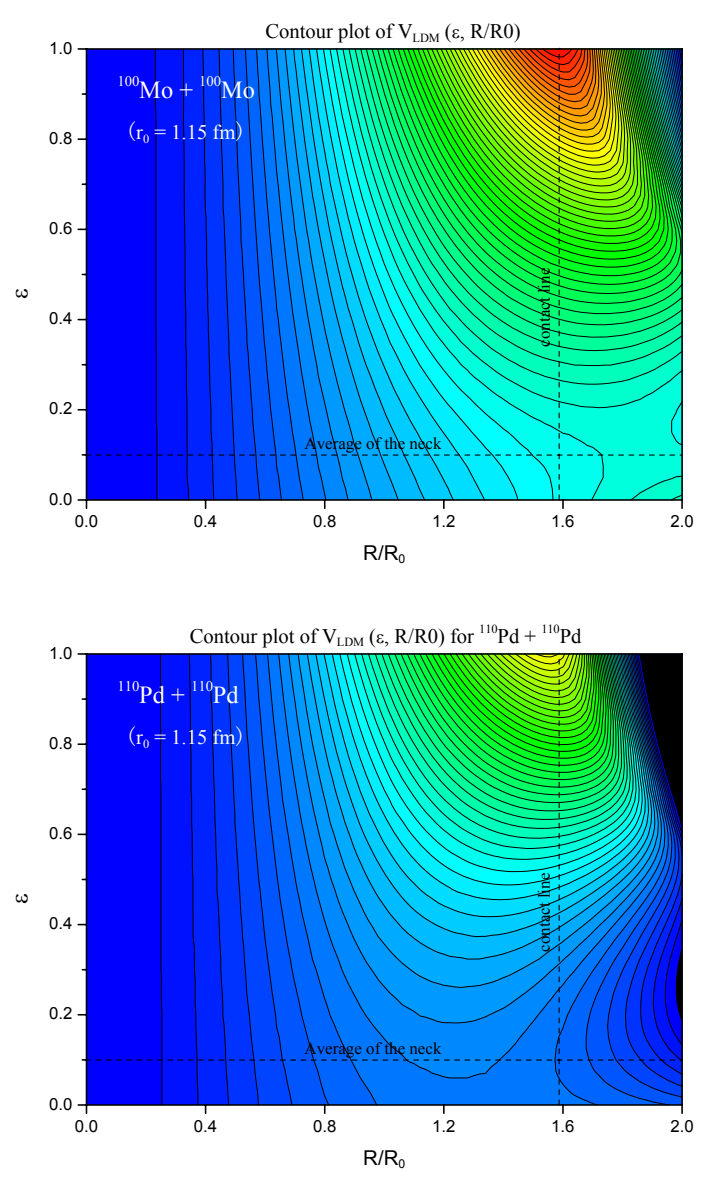

FIG. 3. [Color online] LDM potential map for ${ }^{100} \mathrm{Mo}+{ }^{100} \mathrm{Mo}$ (top) and ${ }^{110} \mathrm{Pd}+{ }^{110} \mathrm{Pd}$ (bottom) as a function of the relative distance and neck parameter.

\section{COUPLING OF THE NECK TO THE OTHER DEGREES OF FREEDOM}

We are now convinced that the denecking process occurs very quickly compared to the typical time scale of the other degrees of freedom determining the fusion. The main argument is based on the potential landscape: the dynamics of the relative distance between the centers of mass of the two nuclei is governed by the diffusion over a potential barrier, which is a slow process [45], whereas the neck is driven by a strong potential slope towards its asymptotic value. But these collective variables are also coupled dynamically through the inertia and friction tensors.

Here, for the sake of simplicity, we only consider two degrees of freedom: the relative distance between the two centers $R$ and the neck $\varepsilon$. This will limit our analysis to symmetric reactions. The formation dynamics can be described by the two-dimension Langevin equation

$$
\gamma\left[\begin{array}{c}
\dot{\varepsilon} \\
\dot{r}
\end{array}\right]=-\left[\begin{array}{c}
\partial V / \partial \varepsilon \\
\partial V / \partial r
\end{array}\right]+\left[\begin{array}{c}
\rho_{1}(t) \\
\rho_{2}(t)
\end{array}\right]
$$


for which we have neglected the inertia term, in order to be consistent with the Smoluchowski approximation. The random force satisfies the fluctuation-dissipation theorem,

$$
\left\langle\rho_{i}(t) \rho_{j}\left(t^{\prime}\right)\right\rangle=2 T \gamma_{i j} \delta\left(t-t^{\prime}\right)
$$

In this equation, $r$ is a dimensionless variable defined as $r=R / R_{0}, R_{0}$ being the radius of the compound nucleus.

We will assume here that around the saddle, the friction tensor $\gamma$ is independent of $r$ and $\varepsilon$. The potential map is such as it has a $U$ shape for the neck variable and a barrier shape for the radial one. Then, the fast neck dynamics could be approximately studied as follow,

$$
\begin{aligned}
\dot{\varepsilon} & =-\left[\gamma^{-1}\right]_{\varepsilon \varepsilon} \frac{\partial V}{\partial \varepsilon}-\left[\gamma^{-1}\right]_{r \varepsilon} \frac{\partial V}{\partial r}+r_{\varepsilon}(t) \\
& \simeq-\left[\gamma^{-1}\right]_{\varepsilon \varepsilon} \frac{\partial V}{\partial \varepsilon}-\left.\left[\gamma^{-1}\right]_{r \varepsilon} \frac{\partial V}{\partial r}\right|_{r=r_{0}}+r_{\varepsilon}(t) .
\end{aligned}
$$

We will assume further that $r_{0}$ is close to the saddle point and $\left|\frac{\partial V}{\partial \varepsilon}\right|_{\varepsilon=\varepsilon_{0}} \gg\left|\frac{\partial V}{\partial r}\right|_{r=r_{0}}$. Then, we can neglect the second term of the r.h.s. of Eq. (10),

$$
\dot{\varepsilon} \simeq-\left[\gamma^{-1}\right]_{\varepsilon \varepsilon} \frac{\partial V}{\partial \varepsilon}+r_{\varepsilon}(t)
$$

Once the neck has reached its asymptotic value, the large confinement potential confines the neck variable.

The differential equation governing the evolution of $r$,

$$
\gamma_{r \varepsilon} \dot{\varepsilon}+\gamma_{r r} \dot{r}=-\frac{\partial V}{\partial r}+\rho_{2}(t)
$$

should be studied on two time scales: first, during the quick evolution of the neck variable, the average value can be approximated by

$$
\gamma_{r \varepsilon}\langle\dot{\varepsilon}\rangle+\gamma_{r r}\langle\dot{r}\rangle \simeq 0
$$

which means that

$$
\Delta\langle r\rangle \simeq-\frac{\gamma_{r \varepsilon}}{\gamma_{r r}} \Delta\langle\varepsilon\rangle
$$

Here, we have also neglected the term $\frac{\partial V}{\partial r}$ because during this transient regime $\frac{\partial V}{\partial \varepsilon}$ is dominating. The initial variance of the relative distance is also related to the final variance of the neck variable corresponding to the Boltzmann distribution:

$$
\left\langle\delta r^{2}(0)\right\rangle=\frac{\gamma_{r \varepsilon}^{2}}{\gamma_{r r}^{2}}\left\langle\delta \varepsilon^{2}(\infty)\right\rangle
$$

Then, once the neck is confined, i.e. $\dot{\varepsilon} \simeq 0$, one has

$$
\gamma_{r r} \dot{r} \simeq-\frac{\partial V}{\partial r}+\rho_{2}(t)
$$

which is a simple one dimension Langevin equation for the relative distance only, with an initial condition that is shifted according to equations (14) and (15).
This approximate dynamical evolution can be checked on a simple test case based on a harmonic potential that is not meant to be realistic,

$$
V(\varepsilon, r)=V_{s}+\frac{1}{2} g \varepsilon^{2}-\frac{1}{2} h\left(r-r_{s}\right)^{2},
$$

for which the coupled differential equations (7) can be exactly solved [47]. With such a potential, the approximate evolution of $r$ is characterized by,

$$
\left\langle r(t)-r_{s}\right\rangle=\left(\left(r_{0}-r_{s}\right)+\frac{\gamma_{r \varepsilon}}{\gamma_{r r}}\left(\varepsilon_{0}-\varepsilon_{\infty}\right)\right) \exp \left[\frac{h t}{\gamma_{r r}}\right]
$$

and

$$
\left\langle\delta r^{2}(t)\right\rangle=\frac{T}{h}\left(\exp \left[\frac{2 h t}{\gamma_{r r}}\right]-1\right)+\left\langle\delta r^{2}(0)\right\rangle \exp \left[\frac{2 h t}{\gamma_{r r}}\right] .
$$

Fig. 4 shows the comparison of this result with the exact solution given in Appendix A and the uncoupled case $\left(\gamma_{r \varepsilon}=0\right)$ for the average trajectory and the fusion probability,

$$
\begin{aligned}
P(t) & =\int_{-\infty}^{r_{s}} \exp \left[-\frac{(r-\langle r(t)\rangle)^{2}}{2 \delta r^{2}(t)}\right] \frac{d r}{\sqrt{2 \pi \delta r^{2}(t)}} \\
& =\frac{1}{2} \operatorname{erfc}\left[\frac{\left\langle r(t)-r_{s}\right\rangle}{\sqrt{2 \delta r^{2}(t)}}\right] .
\end{aligned}
$$

Here we took $\varepsilon_{\infty}=0$ as given by the Boltzmann distribution. The approximate solution of equations $(18,19,21)$ agrees quite nicely with the exact solution, although $g / h=3$ is quite weak. For larger values of $g / h$, the accuracy is even better. Note that this simple model and its approximation are only valid nearby the saddle.

It appears clearly that the fast evolution of the neck variable allows to study the evolution of the other degrees of freedom separately. In the previous sections, we showed that the potential map is very sensitive to the value of the neck. Here, we find that the dynamical coupling through the dissipation tensor shifts the effective initial value of the relative distance. See Eq. (14).

Initially, the neck is far from its equilibrium value. Its large and fast variation shifts the effective initial value of the relative distance. After this transient regime, the fusion will then follow the path that minimizes the potential with respect to the neck and we can do an adiabatic approximation.

With this simple model, the shift is of the order of few femtometers, which is large enough to have an influence on the hindrance of the fusion. It explains the large difference between the fusion probabilities of the uncoupled case and the approximate or exact coupled case that can be observed on Fig. 4. The shift of the initial value of the relative distance is always positive in this model and enlarges the size of the barrier that has to be crossed to reach the compound shape. The fusion is then more hindered. 

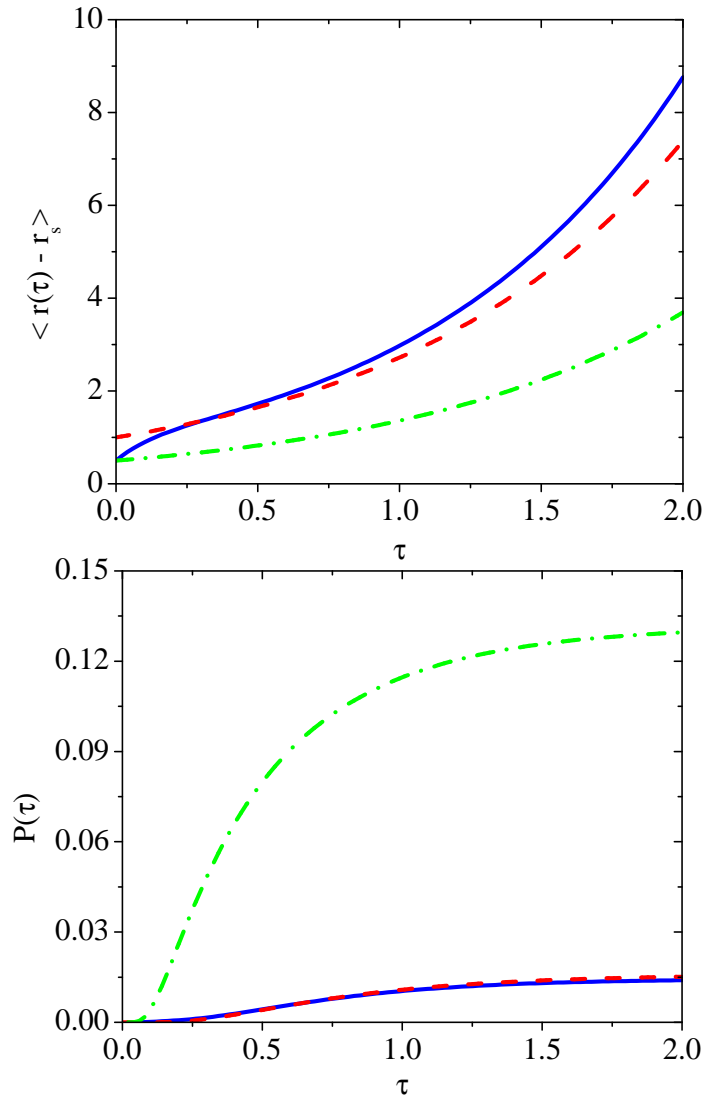

FIG. 4. [Color online] Average trajectory (top) and fusion probability (down) as a function of time for a parabolic potential. The solid blue line represents the exact solution. The green dotted-dashed one represents the uncoupled one $\left(\gamma_{r \varepsilon}=0\right)$. The dashed red curve represents the approximate solution. See text. Here $\gamma_{\varepsilon \varepsilon} / \gamma_{r r}=0.6, \gamma_{r \varepsilon} / \gamma_{r r}=0.5, g / h=3$ and $T / h=0.2$. The time unit is $\gamma_{r r} / h$.

The effect of the shift of the effective injection point on the long time limit of the fusion probability,

$P(t \rightarrow \infty)=\frac{1}{2} \operatorname{erfc}\left[\sqrt{\frac{V\left(\varepsilon_{\infty}, r_{s}\right)-V\left(\varepsilon_{\infty}, r_{0}-r_{s}+\Delta r\right)}{T}}\right]$

is the larger the heavier the system. For systems close to the hindrance border like the ${ }^{110} \mathrm{Pd}+{ }^{110} \mathrm{Pd}, r_{0}$ is close to the saddle and the potential is quite flat. For heavier systems, the potential has a steeper slope near the contact point. Therefore, the shift $\Delta r$ will cause a larger change of the potential barrier for heavier systems.

Of course, the model here is crude: the potential lanscape is simple, we neglected the inertia and we assumed that the friction tensor is constant. It confirms the adiabatic approximation that is usually done in the various models, but it shows that the dynamical coupling be- tween the neck and radial degrees of freedom induces a shift of the effective initial value of the relative distance. We will publish another paper with a more comprehensive study on its magnitude.

Actually, in their so-called "fusion by diffusion" model, Światecki et al [12] introduced an initial shift of the injection point considered to be an adjustable parameter ranging from 0 to $3 \mathrm{fm}$. Here, we propose a justification to it. More recently, Liu et al [48] explore numerically the effect of the non-diagonal term of the friction tensor on the injection point. They conclude that the average injection point is not shifted. This is in contradiction with our results.

\section{CONCLUSION}

Since super-heavy elements are produced in extremely small numbers, their main characteristics are not accessible yet. But, using a fission-evaporation code, it is possible to constrain strongly the shell correction energy of their ground state [33, 49] if we know the fusion probability. Unfortunately, experimental fusion cross sections are not reliable because it is very difficult to distinguish between fission and quasi-fission. Fusion models should then be assessed by other means.

In this article, we have stressed the importance of the neck parameter that can change the fusion cross sections by orders of magnitude. We have shown that the neck degree of freedom evolves faster than the relative distance between the two fusing nuclei. Then the approximation of using an asymptotic value of the neck is justified.

The rapid evolution of the neck parameter changes the potential landscape seen by the other collective variables. The experimental appearance of the hindrance of the fusion for symmetric reactions confirms this conclusion. This rapid evolution of the neck also changes the initial value of the other collective variables through a dynamical coupling. For the relative distance, the shift is not negligible and should be included in the models. Our analysis gives a theoretical justification to the adjustable shift introduced by Świątecki et al [12] in order to reproduce the data. Finally, it is important to note that both effects enlarge the hindrance of the fusion.

This analysis of the influence of the neck dynamics on the fusion of heavy nuclei is mainly based on simplified analytical models and is therefore limited to symmetric reactions. The asymmetry degree of freedom complicates the analysis which cannot be simply handled with analytical toy models. Therefore, a more complete study will be published in another paper.

\section{ACKNOWLEDGMENTS}

We are indebted to Y. Lallouet for helpful remarks. The present work was supported by the JSPS grants No. 18540268, L10708, by the Natural Science Foundation of 
China under grant Nos. 10979024, 10905021, the Key project of Science and Technology Research of Ministry of Education of China under grant No. 209053, and the Natural Science Foundation of Zhejiang province, China under grant No. Y6090210 and by the LIA GANILRIKEN. The authors also acknowledge supports and hospitality by RCNP Osaka Univ., GANIL, Huzhou Teachers College, and IPT, CE-Saclay, which enable us to continue the collaboration.

\section{Appendix A: Exact solution of the diffusion over the parabolic potential}

Following the method of Ref. [47], it is possible to solve exactly the diffusion problem in the overdamped limit on the potential landscape of Eq. (17). The distributions are Gaussian characterized by

$$
\begin{aligned}
\langle\varepsilon(t)\rangle= & \varepsilon_{0} \frac{e^{a_{+} t}+e^{a_{+} t}}{2}+\frac{e^{a_{-} t}-e^{a_{+} t}}{2 \sqrt{\Delta}} \\
& \times\left[2\left(r_{0}-r_{s}\right) \gamma_{r \varepsilon} h+\varepsilon_{0}\left(\gamma_{\varepsilon \varepsilon} h+\gamma_{r r} g\right)\right] \\
\left\langle\delta \varepsilon^{2}(t)\right\rangle= & \frac{T \gamma_{r r}}{\gamma_{r r} \gamma_{\varepsilon \varepsilon}-\gamma_{r \varepsilon}^{2}}\left\{\frac { e ^ { 2 a _ { + } t } - 1 } { 2 a _ { + } \sqrt { \Delta } } \left(\sqrt{\Delta}+2 \frac{h \gamma_{r \varepsilon}^{2}}{\gamma_{r r}}\right.\right. \\
& \left.-\gamma_{\varepsilon \varepsilon} h-\gamma_{r r} g\right)+\frac{e^{2 a_{-} t}-1}{2 a_{-} \sqrt{\Delta}}(\sqrt{\Delta} \\
& \left.\left.-2 \frac{h \gamma_{r \varepsilon}^{2}}{\gamma_{r r}}+\gamma_{\varepsilon \varepsilon} h+\gamma_{r r} g\right)\right\},
\end{aligned}
$$

with

$$
\begin{aligned}
a_{ \pm} & =\frac{\gamma_{\varepsilon \varepsilon} h-\gamma_{r r} g \pm \sqrt{\Delta}}{2\left(\gamma_{r r} \gamma_{\varepsilon \varepsilon}-\gamma_{r \varepsilon}^{2}\right)} \\
\Delta & =\left(\gamma_{\varepsilon \varepsilon} h-\gamma_{r r} g\right)^{2}+4 g h\left(\gamma_{r r} \gamma_{\varepsilon \varepsilon}-\gamma_{r \varepsilon}^{2}\right) .
\end{aligned}
$$

Here the friction tensor is symmetric: $\gamma_{r \varepsilon}=\gamma_{\varepsilon r}$. Without off-diagonal term $\left(\gamma_{r \varepsilon}=0\right)$, these expressions simply become,

$$
\begin{aligned}
\langle\varepsilon(t)\rangle & =\varepsilon_{0} e^{-\frac{g t}{\gamma_{\varepsilon \varepsilon}}} \\
\left\langle\delta \varepsilon^{2}(t)\right\rangle & =\frac{T}{g}\left(1-e^{-2 \frac{g t}{\gamma_{\varepsilon \varepsilon}}}\right) .
\end{aligned}
$$

With the approximation of Eq. (10), we have,

$$
\begin{aligned}
\langle\varepsilon(t)\rangle \simeq & -\frac{\gamma_{r \varepsilon}}{\gamma_{r r}} \frac{h}{g}\left(r_{0}-r_{s}\right)\left(1-e^{-g\left[\gamma^{-1}\right]_{\varepsilon \varepsilon} t}\right) \\
& +\varepsilon_{0} e^{-g\left[\gamma^{-1}\right]_{\varepsilon \varepsilon} t} \\
\left\langle\delta \varepsilon^{2}(t)\right\rangle \simeq & \frac{T}{g}\left(1-e^{-2 g\left[\gamma^{-1}\right]_{\varepsilon \varepsilon} t}\right) .
\end{aligned}
$$

Then, for long times, we take the average value given by the Botzmann distribution as an asymptotic value of the neck variable, $\langle\varepsilon(t \rightarrow \infty)\rangle=\varepsilon_{\infty}$.

Similarly, for $r$, the exact solution is characterized by

$$
\begin{aligned}
\langle r(t)\rangle= & r_{s}+\left(r_{0}-r_{s}\right) \frac{e^{a_{+} t}+e^{a_{+} t}}{2}+\left[2 \varepsilon_{0} \gamma_{r \varepsilon} g\right. \\
& \left.+\left(r_{0}-r_{s}\right)\left(\gamma_{\varepsilon \varepsilon} h+\gamma_{r r} g\right)\right] \frac{e^{a_{+} t}-e^{a_{-} t}}{2 \sqrt{\Delta}} \\
\left\langle\delta r^{2}(t)\right\rangle= & \frac{T \gamma_{\varepsilon \varepsilon}}{\gamma_{r r} \gamma_{\varepsilon \varepsilon}-\gamma_{r \varepsilon}^{2}}\left\{\frac { e ^ { 2 a _ { + } t } - 1 } { 2 a _ { + } \sqrt { \Delta } } \left(\sqrt{\Delta}-2 \frac{g \gamma_{r \varepsilon}^{2}}{\gamma_{\varepsilon \varepsilon}}\right.\right. \\
& \left.+\gamma_{\varepsilon \varepsilon} h+\gamma_{r r} g\right)+\frac{e^{2 a_{-} t}-1}{2 a_{-} \sqrt{\Delta}}(\sqrt{\Delta} \\
& \left.\left.+2 \frac{g \gamma_{r \varepsilon}^{2}}{\gamma_{\varepsilon \varepsilon}}-\gamma_{\varepsilon \varepsilon} h-\gamma_{r r} g\right)\right\} .
\end{aligned}
$$

When the two variables are uncoupled $\left(\gamma_{r \varepsilon}=0\right)$, these expressions simply become,

$$
\begin{aligned}
\langle r(t)\rangle & =r_{s}+\left(r_{0}-r_{s}\right) e^{\frac{h t}{\gamma_{r r}}} \\
\left\langle\delta r^{2}(t)\right\rangle & =\frac{T}{h}\left(e^{2 \frac{h t}{\gamma_{r r}}}-1\right) .
\end{aligned}
$$

The approximate solution is characterized by Eqs $(18,19)$.
[1] C.-C. Sahm, H.-G. Clerc, K.-H. Schmidt, W. Reisdorf, P. Armbruster, F.P. Heßberger, J.G. Keller, G. Münzenberg and D. Vermeulen, Z. Phys. A319 (1984) 113

[2] K.-H. Schmidt and W. Morawek, Rep. Prog. Phys. 54 (1991) 949

[3] W. J. Świątecki, Nucl. Phys. A376 (1982) 275

[4] G. Royer and B. Remaud, Nucl. Phys. A444 (1985) 477

[5] J.P. Błocki, H. Feldmeier and W. J. Świątecki, Nucl. Phys. A459 (1986) 145

[6] Y. Abe, D. Boilley, G. Kosenko, J.D. Bao, C.W. Shen, B.G. Giraud and T. Wada, Prog. Theor. Phys. Suppl. 146 (2002) 104

[7] Y. Abe, C.W. Shen, G.I. Kosenko and D. Boilley, Phys. At. Nucl. 66 (2003) 1057

[8] C. Shen, G. Kosenko and Y. Abe, Phys. Rev. C66 (2002) $061602(\mathrm{R})$

[9] C. Shen, Y. Abe, Q. Li and D. Boilley, Sciences in China

\section{G52 (2009) 1458}

[10] W. J. Świątecki, K. Siwek-Wilczyńska and J. Wilczyński, Acta Phys. Pol. B34 (2003) 2049

[11] W. J. Świątecki, K. Siwek-Wilczyńska and J. Wilczyński, Int. J. Mod. Phys. E13 (2004) 261

[12] W. J. Świątecki, K. Siwek-Wilczyńska and J. Wilczyński, Phys. Rev. C71 (2005) 014602

[13] Y. Aritomo and M. Ohta, Nucl. Phys. A744 (2004) 3

[14] Y. Aritomo, Nucl. Phys. A780 (2006) 222

[15] V.I. Zagrebaev, Phys. Rev. C64 (2001) 034606

[16] V. Zagrebaev and W. Greiner, J. Phys. G 31 (2005) 825

[17] D. Hinde et al, contribution to the Fusion11 conference, to appear in EPJ web of conferences.

[18] K. Siwek-Wilczyńska, E. Siemaszko and J. Wilczyńsk, Acta Phys. Pol. B33 (2002) 451

[19] K. Siwek-Wilczyńska and J. Wilczyński, Phys. Rev C69 (2004) 024611 
[20] N. Rowley, N. Grar and K. Hagino, Phys. Lett. B632 (2006) 243

[21] K. Washiyama, D. Lacroix and S. Ayik, Phys. Rev. C79, (2009) 024609

[22] C.M. Ko, Z. Phys. A286 (1978) 405

[23] A. Diaz-Torres, D.J. Hinde, M. Dasgupta, G.J. Milburn and J.A. Tostevin, Phys. Rev. C78 (2008) 064604

[24] S. Yusa, K. Hagino and N. Rowley, Phys. Rev. C82 (2010) 024606

[25] C.E. Aguiar, V.C. Barbosa, L.F. Canto and R. Donangelo, Nucl. Phys. A472 (1987) 571

[26] A. Iwamoto and K. Harada, Z. Phys. A326 (1987) 201

[27] J.D. Bao, Int. J. Mod. Phys. E15 (2006) 1625

[28] G.G. Adamian, N.V. Antonenko, A. Diaz-Torres and W. Scheid, Nucl. Phys. A671 (2000) 233

[29] R. Moustabchir and G. Royer, Nucl. Phys. A683 (2001) 266

[30] G. Royer, K. Zbiri and C. Bonilla, Nucl. Phys. A 730 (2004) 355

[31] Y. Abe, C.W. Shen, G. Kosenko, D. Boilley and B. Giraud, Int. J. Mod. Phys. E17 (2008) 2214

[32] Y. Abe, C.W. Shen, D. Boilley and B. Giraud, Int. J. Mod. Phys. E18 (2009) 2169

[33] D. Boilley, Y. Abe, C.W. Shen and B. Yılmaz, CERNProceedings-2010-001 (2010) 479

[34] D. Boilley, C. Shen, Y. Abe and B.G. Giraud, to appear in the EPJ Web of Conferences

[35] K. Zhao, Z. Li, X. Wu and Z. Zhao, Phys. Rev. C79 (2009) 024614

[36] Z.-H. Liu and J.-D. Bao, Phys. Rev. C81 (2010) 044606

[37] K. Sato, A. Iwamoto, K. Harada, S. Yamaji and S. Yoshida, Z. Phys. A288 (1978) 383

[38] J. Blocki, Y. Boneh, J. R. Nix, J. Randrup, M. Robel, A. J. Sierk and W. J. Swiatecki, Ann. Phys. 113 (1978) 330

[39] T. Wada, Y. Abe and N. Carjan, Phys. Rev. Lett. 70 (1993) 3538

[40] M. v. Smoluchowski, Physik. Zeit. 17 (1916) 585

[41] M. Mason and W. Weaver, Phys. Rev. 23 (1924) 412

[42] S. Chandrasekar, Rev. Mod. Phys. 15 (1943) 1

[43] B.U. Felderhof, J. Stat. Phys. 109 (2002) 483

[44] H. Minowa and N. Mishima, Lettere Al Nuovo Cimento 37 (1983) 99

[45] D. Boilley, Y. Abe and J.D. Bao, Eur. Phys. J. A18 (2003) 627

[46] C. Shen, D. Boilley, Q. Li, J. Shen and Y. Abe, Phys. Rev. C83 (2011) 054620

[47] Y. Abe, D. Boilley, B.G. Giraud and T. Wada, Phys. Rev. E61 (2000) 1125

[48] Zu-Hua Liu and Jing-Dong Bao, Phys. Rev. C83 (2011) 044613

[49] A. Marchix, PhD thesis, Université de Caen (2007), http://tel.archives-ouvertes.fr/tel-00197012/ 